\title{
Predicting the effective thermal conductivity of geo-materials using artificial neural networks
}

\author{
Dinesh Shrestha ${ }^{1, *}$, and Frank Wuttke ${ }^{1}$ \\ ${ }^{1}$ Marine and Land Geomechanics and Geotechnics, Kiel University, Kiel, Germany
}

\begin{abstract}
Soil thermal conductivity is an important thermal property used in heat transfer modelling and geo-energy applications. Because of its complex nature and depending on several factors such as porosity, moister content, structure, etc., it is always challenging to predict the thermal conductivity of geo-materials. In the past, many predictions models like theoretical, semi-empirical, empirical models have been proposed based on the experimental data. However, these models are more specific to certain boundary conditions. Therefore, in this study, an artificial neural network (ANN) approach was used to predict the thermal conductivity of geo-materials as a function of porosity, gradation and mineralogy. A comparison between existing prediction models and the developed ANN model for predicting thermal conductivity is also given.
\end{abstract}

\section{Introduction}

Heat transfer in soils is an important issue in many geoenergy applications, such as high voltage underground cable, radioactive waste disposal, heat pumps and energy piles. The performance and efficiency of these applications depend on the thermal properties of the geomaterials where they built. A knowledge of the soil thermal properties is thus essential to understand the process of heat transfer in soils and designing such applications. Soil thermal properties mainly consists of thermal conductivity, diffusivity and specific heat capacity. Among them, thermal conductivity $(\lambda)$ is a very important property in heat transfer modelling and geomaterials design.

Since thermal conductivity of soil is function of moisture content, density, mineralogy, gradation and other environmental external factors, it is always challenging to predict soil thermal conductivity. Many researches in the past decades have been performed to quantify the effects of various factors on thermal conductivity of different soils and to correlate the thermal conductivity with those influence factors [1-5]. Earlier researchers [5-8] have put more effort on developing models based on basic geotechnical index properties. Like Cote \& Konrad [5] indicated that effects of grain mineralogy and fabric should be considered while predicting thermal conductivity of soils. So, basically three types of approach such as analytical/theoretical, empirical/semi-empirical and numerical have been in use to predict the thermal conductivity of soils. The analytical models are more complex which are adopted from other physical models and involve more calculation parameters. In contrast, empirical models are mostly developed from experimental data regression. These models are however more specific to certain boundary conditions and specific soil types and are unable to predict for special geomaterials developed for buried power cables [9,10]. Shrestha $[9,10]$ developed the high thermal conductivity backfill materials by changing gradation and adding fine materials to natural sand. He stated that the existing prediction models were unable to capture these changes and the models need to be developed to predict these characteristics. It can be said that the prediction model that is simple in application, accurate in prediction result and applicable for all types of soil is needed for assessing the thermal conductivity of soils effectively. Therefore, artificial neural network (ANN) as a novel approach is proposed in this study to predict the soil thermal conductivity of geomaterials.

ANN has been successfully used in solving many engineering problems. Shahin [11] predicts settlement of shallow foundations on granular soils using ANN and found better prediction than traditional method. Other researchers predict liquefaction potential of soil [12], permeability of clay liners [13], thermal conductivity of dry granular media [14], electrical resistivity of soils [15]. The details about ANN is explained in chapter 2 .

In this study, a detailed modelling work is performed to develop thermal conductivity prediction models for modified geomaterials (shrestha et al) from influence factors such as porosity (or density), mineralogy (quartz content) and gradation. The predicted results from ANN model are verified via the cross-validation method. The developed model is also compared with existing prediction models. The prediction performance of ANN model with semi-empirical prediction models is evaluated by performance indices such as MAE, RMSE.

\footnotetext{
* Corresponding author: dinesh.shrestha@ifg.uni-kiel.de
} 


\section{Artificial neural networks}

Artificial neural networks (ANN) are form of artificial intelligence (AI) which simulate the behaviour of human brain and nervous system. ANNs consist basically of three layers viz. input layer, hidden layer and output layer. Each layer is interconnected by processing elements like cells in brain known as neurons. The neurones are typically organised into the layers. A typical architecture of feed-forward multi-layer ANNs is shown in Fig.1. where $X_{i}$ represents input parameters, $Y_{i}$ $\& Y_{j}$ are the neurones in hidden layer and $Z$ is the output in output layer. A connection medium, which connect each neuron in given layer to all the neurons in next layers, is known as weight. This connection has tendency to deliver the calculation results to next layer completely. A network with single hidden layer is called shallow network whereas with multiple layer is called deep neural network. The selection of hidden layers and neurons is basically trial and error but sometimes depends on complexity of the problems. Previous studies recommend one or two hidden layers are enough for solving most civil engineering problems [16].

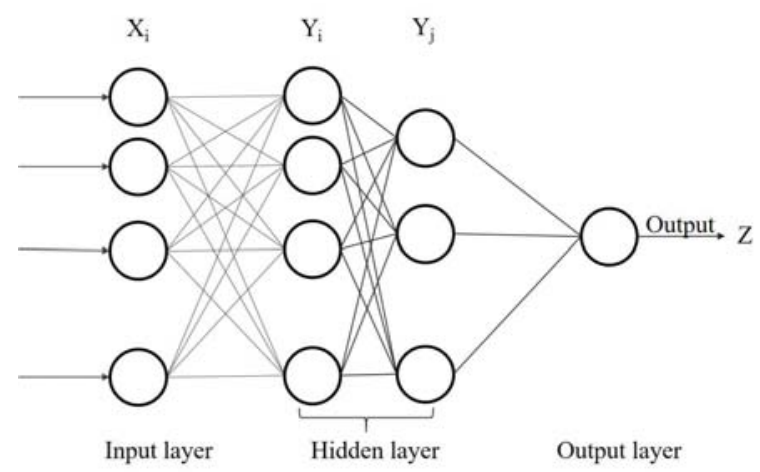

Fig. 1. Schematic diagram of ANN.

The backpropagation learning algorithm, which is one of the most popular algorithms in ANNs, has been extensively and successfully used to simulate many engineering problems like settlements, permeability, landslide stabilization [17]. The input parameters will be processed in neurons to get the outputs by using following equations:

$$
Y_{\mathrm{i}}=\sum F_{i}\left(W_{i} X_{i}\right)+b_{i}
$$

where $\mathrm{W}$ is the weight connecting neurons, $\mathrm{b}$ is bias of neuron and $\mathrm{F}$ is activation function of neuron. Repeating above steps and updating the weights until the desired outputs are reached. The result of neural network is output in output layer. A well-trained ANN model should have a coefficient of determination, $\mathrm{R}^{2}$, is 1 or very close to 1 and other errors should be very low.

\subsection{Development of ANN}

ANNs learn and train the data given to them and update the weights to find relationship between model inputs and corresponding outputs. This process is called learning or training. Once this phase of the model has been successfully accomplished, the performance of the trained model must be validated using an independent validation data set. The cross-validation technique the most appropriate method for developing ANN models was used in this study as stopping criteria to avoid overfitting. In this method, the data was divided into three subsets; training, validation and testing set but it keeps changing the datasets. It means it repeats the division of the data instead of retaining the initially divided sets. The reason for doing this is to avoid overfitting of the model. The training set is used to update the weights and the updating process is monitored by the error of validation data. The training process will stop, once the error of validation data in the validation set begin to increase. The model generalization is considered to reach its best phase at this point. The testing set is utilized to evaluate the performance of trained network. In this study, the database was first divided into two parts; 1) training data, 2) testing data. The latter data will be used to assess the performance of developed ANN model based on performances indices RMSE, MAE. These data will not be used to develop the ANN models. The first data was further divided into training set, validation set, and testing set in the ratio of 70:15:15 to develop the ANN models.

The training, validation and testing processes of multi-layer perceptions (MLPs) was conducted in the artificial neural network toolbox in MATLAB 2019, which is a popular numerical computation and visualization software. The Levenberg-Marquard (LM) backpropagation learning algorithm which is fast and efficient algorithm was used with one hidden layer since one hidden layer with enough neurons can approximate any continuous function in geotechnical engineering. The optimum number of neurons was determined by varying their numbers from 1 to the numbers at which the network reaches its well-trained point. The activation function used in each neural network is Log-sigmoid and linear in hidden and output layers, respectively. Two momentum factors, $\mu, 0.01$ and 0.001 were set for the ANN models to get best structure in each neural network. The maximum epochs (training cycles) were set as 1000 times in each ANN model. The performance of each developed model was evaluated by determining coefficient of determination, $\mathrm{R}^{2}$ and MSE. The $\mathrm{R}^{2}$ is determined for training, testing and validation set and MSE is calculated for independent testing data to check the performance of proposed model. If $\mathrm{R}^{2}$ are close to 1 and MSE is almost 0.005, the developed model is considered as well-trained and best model. The optimal configuration of ANN model with R2 $=0.98$ and MSE $=$ 0.008 is 8 neurons in the hidden layer and 0.001 momentum factor. Two further parameters, RMSE and MAE were also used to compare the proposed model and existing thermal conductivity prediction models.

$$
\begin{aligned}
& \mathrm{RMSE}=\sqrt{ } 1 / \mathrm{N} \sum\left(\lambda_{\mathrm{mi}}-\lambda_{\mathrm{pi}}\right)^{2} \\
& \mathrm{MAE}=\sum\left|\lambda_{\mathrm{m}}-\lambda_{\mathrm{p}}\right| / \mathrm{N}
\end{aligned}
$$

where $\lambda_{\mathrm{m}}$ is measured thermal conductivity, $\lambda_{\mathrm{p}}$ predicted thermal conductivity and $\mathrm{N}$ is the number of sample. 


\subsection{Database creation}

The database was created from the literature. The dry thermal conductivity data obtained from the experiment for developed geomaterials [9] were collected to develop ANN model. Three kinds of sands, sand-A, sand-B and sand-C were used to develop high conductive materials by modifying natural gradation to fuller gradation and adding fine content with different proportion. The measurement technique to measure the thermal conductivity of the materials in lab used was transit needle probe method. The details about the material development and measurement can be found in Shrestha et.al $[9,10]$. Four input parameters, porosity, quartz content, gradation parameters such as coefficient of curvature $\left(\mathrm{C}_{-} \mathrm{c}\right)$ and coefficient of uniformity $\left(\mathrm{C}_{-} \mathrm{u}\right)$ were selected as input parameters. As the natural sands were modified by changing gradation, incorporating gradation parameters could be promising idea to get best results. The $\mathrm{C}_{-} \mathrm{u}$ for fuller gradation is 35 and C_c for $8 \mathrm{mmF}$, $4 \mathrm{mmF}$, and $2 \mathrm{mmF}$ are $1.98,2.72$ and 2,58 respectively. The quartz contain for sand-A, sand-B and sand-C are $99 \%, 80 \%$ and $70 \%$ respectively.

\section{Thermal conductivity prediction models}

Previous studies suggested different prediction models for dry thermal conductivity., theoretical and empirical models. Existing theoretical models, listed in Table 1, have been proposed for two-phase materials based on the properties of each phase, geometry, and the volumetric fractions (or porosity). However, these models are unable to quantify the microstructure effect, which is strongly affecting thermal conductivity of dry soils due to the higher ratio of thermal conductivity between solid particles and air $(\lambda \mathrm{s} / \lambda \mathrm{a}>100)$.

Table 1. Theoretical thermal conductivity models for dry soils

\begin{tabular}{|c|c|}
\hline Model & Equation \\
\hline Series [2] & $\lambda_{d}=\left[\frac{n}{\lambda_{a}}+\frac{1-n}{\lambda s}\right]^{-1}$ \\
\hline Parallel [2] & $\lambda_{d}=n \lambda_{a}+(1-n) \lambda_{s}$ \\
\hline $\begin{array}{c}\text { Geometric } \\
\text { mean [2] }\end{array}$ & $\lambda_{d}=\lambda_{a}{ }^{n} \lambda_{s}{ }^{(1-n)}$ \\
\hline $\begin{array}{l}\text { Hashin and } \\
\text { Shtrikman } \\
\text { Boundary [18] }\end{array}$ & $\begin{array}{l}\lambda_{d U}=\lambda_{s}+\frac{3 n \lambda_{s}\left(\lambda_{a}-\lambda_{s}\right)}{3 \lambda_{s}+(1-n)\left(\lambda_{a}-\lambda_{s}\right)} \\
\lambda_{d L}=\lambda_{a}+\frac{3(1-n) \lambda_{a}\left(\lambda_{s}-\lambda_{a}\right)}{3 \lambda_{s}+n\left(\lambda_{s}-\lambda_{a}\right)}\end{array}$ \\
\hline $\begin{array}{c}\text { Cubic Cell } \\
{[19]}\end{array}$ & $\begin{array}{l}\lambda_{d}=\left[\frac{\beta-1}{\lambda_{a} \beta}+\frac{\beta}{\lambda_{a}\left(\beta^{2}-1\right)+\lambda_{s}}\right]^{-1} \\
\beta=\left[\frac{1}{1-n}\right]^{1 / 3}\end{array}$ \\
\hline Maxwell [20] & $\lambda_{d}=\lambda_{s}\left[\frac{\lambda_{a}+2 \lambda_{s}+2 n\left(\lambda_{a}-\lambda_{s}\right)}{\lambda_{a}+2 \lambda_{s}-n\left(\lambda_{a}-\lambda_{s}\right)}\right]$ \\
\hline
\end{tabular}

$\lambda_{d}=$ dry thermal conductivity; $n=$ porosity; $\lambda_{a}=$ thermal conductivity of air; $\lambda_{s}=$ thermal conductivity of solid particles
Fig. 2 presents comparison of measured thermal conductivity values with theoretical models which are computed assuming that the thermal conductivity of quartz and air are $\lambda_{\text {mineral }}=7.7 \mathrm{~W} \mathrm{~m}^{-1} \mathrm{~K}^{-1}$ and $\lambda_{\text {air }}=0.024$ $\mathrm{W} \mathrm{m}{ }^{-1} \mathrm{~K}^{-1}$ at $20{ }^{\circ} \mathrm{C}$. As seen in Fig. 2, the thermal conductivity decreases sharply from that of quartz. As expected, all measured thermal conductivity values lie between Weiner bound (series and parallel flow model) and Hashin and Shtrikman Bound [18]. Geometric mean method overestimates the thermal conductivity of all studied mixes while Cubic cell [19] underestimates the measured thermal conductivity. Geometric mean method only gives satisfactory results when the ratio of thermal conductivity of solids to that of fluids (either liquid or gas), $\lambda_{\mathrm{s}} / \lambda_{\mathrm{f}}<15$. In this case, air being fluid, $\lambda_{\mathrm{s}} / \lambda_{\mathrm{f}}>100$ leads to overestimation of thermal conductivity. Maxwell model [20] is identical with Hashin and Shtrikman upper boundary model. From this comparison, it can be said that the dry thermal conductivity considers inherent presence of particle contacts along with volume fraction and bulk conductivity of each constituent.



Fig. 2. Comparison of experimental results with theoritical models.

Only few empirical models, listed in Table 2, have been developed to estimate dry thermal conductivity for different types of soil according to soil fabric, gradation, mineral thermal conductivity and porosity or dry density. These models are developed from the regression of the experimental data.

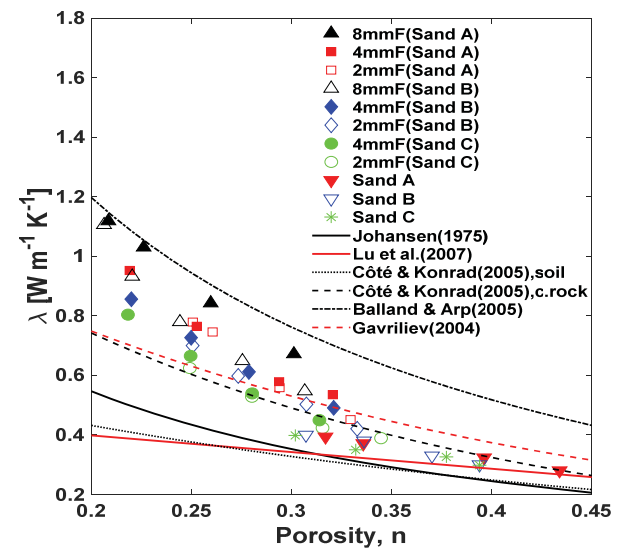

Fig. 3. Comparison of experimental results with semiempirical models. 
Table 2. Semi-empirical $\lambda$ models for dry soils

\begin{tabular}{|c|c|c|}
\hline Model & Equation & $\begin{array}{l}\text { Boundary } \\
\text { Condition }\end{array}$ \\
\hline $\begin{array}{l}\text { Johansen, } \\
1975 \text { [3] }\end{array}$ & $\begin{array}{l}\lambda_{d}=\frac{0.135 \rho_{d} 164.7}{\rho_{s}-0.947 \rho_{d}} \\
\lambda_{d}=0.039 n^{-2.2)}\end{array}$ & $\begin{array}{l}\text { For natural soils } \\
\text { For crushed } \\
\text { rocks }\end{array}$ \\
\hline $\begin{array}{l}\text { Côté \& } \\
\text { Konrad, } \\
2005 \text { [5] }\end{array}$ & $\lambda_{d}=\chi 10^{-\eta n)}$ & $\begin{array}{l}\text { For natural soils } \\
\chi=0.75, \eta= \\
1.20 \\
\text { For } \\
\text { rocks } \\
\chi=1.7, \eta=1.8 \\
\text { For orushed } \\
\text { fibrous soils } \\
\chi=0.30, \eta= \\
0.87\end{array}$ \\
\hline $\begin{array}{l}\text { Lu, } 2007 \\
{[6]}\end{array}$ & $\lambda_{d}=0.56 n+0.51$ & $\begin{array}{l}\text { For mineral soils } \\
0.2<n<0.6\end{array}$ \\
\hline $\begin{array}{l}\text { Balland } \\
\& \quad \text { Arp, } \\
2005[21]\end{array}$ & $\lambda_{d}=\frac{\left(a \lambda_{s}-\lambda_{a}\right) \rho_{b}+\lambda_{a} \rho_{s}}{\rho_{s}-(1-a) \rho_{h}}$ & $a=0.053$ \\
\hline $\begin{array}{l}\text { Gavriliev, } \\
2004 \text { [22] }\end{array}$ & $\begin{array}{r}\lambda_{d}=0.025+0.0238 \rho_{d} \\
-0.193 \rho_{d}^{2}+0.114 \rho_{d}^{3}\end{array}$ & $\begin{array}{l}\text { For mineral soils } \\
\rho_{d}<2 \mathrm{~g} / \mathrm{cc}\end{array}$ \\
\hline
\end{tabular}

$\lambda_{d}=$ dry thermal conductivity; $n=$ porosity; $\rho_{s}=$ density of solid particles; $\rho_{d}=$ dry density ; $\rho_{b}=$ bulk density

Fig. 3 shows measured thermal conductivity values with semi-empirical thermal conductivity models against porosity. All the models for soil underestimate the thermal conductivity values of modified sands besides Balland \& Arp model. However, Gavriliev model [22] predicts the thermal conductivity for porosities greater than 0.25. Nevertheless, these models show good agreement with measured thermal conductivity of natural sand. The fact is that the models are mainly based on porosity (or dry density) of media and don't consider inherent presence of contacts quality in the medium. Therefore, ANN as a new approach is used in this study to capture this behaviour.

\section{Results and discussion}

A comparison of predicted thermal conductivity obtained from ANN models developed with measured thermal conductivity is shown in Fig. 4 for two data sets training and testing. The second data set was completely used for checking the performance of the developed ANN model. These data were not used for developing models. The first data set includes $70 \%$ training, $15 \%$ validation and $15 \%$ testing set. performances based on these performance indices. The cross-validation technique is effective and feasible for developing ANN models as there is good agreement between predicted and measured values in all subsets (training, validation and testing). It is observed from the figure that the predicted thermal conductivity values are quite close to the measured data, since their $\mathrm{R}^{2}$ values are close to 0.98 (all), which indicates high quality of ANN structure. The other performance indices, RMSE, MAE calculated for the developed ANN models are presented in Table 3.

A comparison between ANN models and semiempirical models listed 2 has been also made to assess the performance of developed ANN models. The results are shown in Fig. 5. It is evident that the ANN model predicts much better than semi-empirical models. Among the models, Gavriliev [22] model predicts well upto the porosity of 0.25 . Statistical analysis was performed to quantitively assess the accuracy of the proposed ANN models and semi-empirical models. The RMSE and MAE were calculated to determine the accuracy of the models. They are presented in Table 4 . The analysis states that the developed ANN model provides best matching to the measured data of modified geomaterials as it possesses the lowest RMSE and MAE values (Table 3 ).

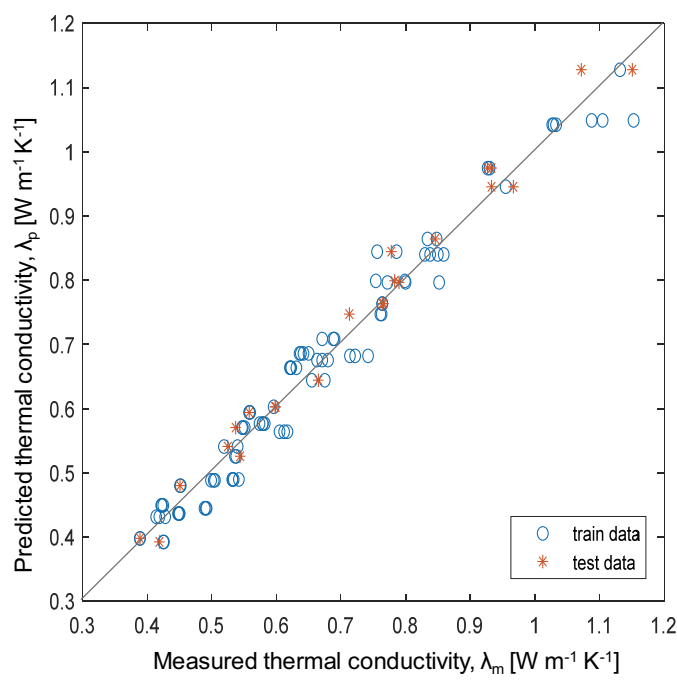

Fig. 4. Comparison of predicted thermal conductivity from ANN model with measured conductivity. The test data in the figure are not used for developing ANN model.

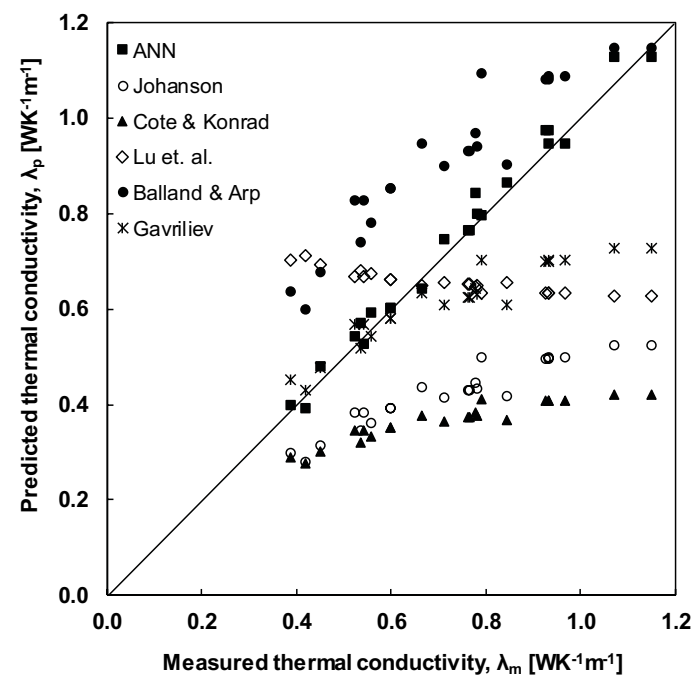

Fig. 5. Comparison of calculation performance between ANN models and semi-empirical models for modified geomaterials. 
Table 3. Semi-empirical $\lambda$ models for dry soils

\begin{tabular}{|c|c|c|}
\hline Model & RMSE & MAE \\
\hline ANN model & 0.0292 & 0.0237 \\
\hline Cote \& Konrad & 0.3976 & 0.3605 \\
\hline Johansen & 0.3365 & 0.3054 \\
\hline Lu et. al. & 0.2376 & 0.2006 \\
\hline Gavriliev & 0.1739 & 0.1303 \\
\hline Balland \& Arp & 0.2039 & 0.1892 \\
\hline
\end{tabular}

\section{Conclusions}

In this study, ANN model is developed to predict the thermal conductivity of modified geomaterials which have high thermal conductivity in dry state due to change in gradation. The models have been also verified and compared with existing semi-empirical models to reinforce their applicability. The semi-empirical models are primarily based on porosity. That's why these models couldn't capture the improvement of thermal conductivity due to improvement contact quality and number between grains because of modification of gradation. The developed models able to capture the heat transfer behavior through grains to grains in densely packed backfill soils. The incorporating of gradation parameters such as $\mathrm{C}_{-} \mathrm{u}$ and $\mathrm{C}_{-} \mathrm{c}$ and quartz contact results a very well-trained ANN models. The proposed ANN model has shown high prediction performance based on the performance's indices.

\section{References}

1. D.A. de Vries, Physics of plant environment, In W.R. Van Wijk (eds.), 210-235. Amsterdam: NorthHolland Publ. Co. (1963)

2. O.T. Farouki, CRREL Monograph 81-1, US Army Corps of Engineers, Cold Regions Research and Engineering Laboratory, Hanover, N.H. (1981)

3. O. Johansen, Ph.D. diss. Norwegian Univ. of Science and Technol, Thermal conductivity of soils. Trondheim (1975)

4. M.V.B.B. Gangadhara Rao \& D.N. Singh, Can. Geotech. J. 36: 767-773 (1999)

5. J. Côté \& J.M. Konrad, Can. Geotech. J. 42: 443458 (2005a)

6. S. Lu, T. Ren, Y. Gong \& R. Horton, Soil Sci. Soc. Am. J. 71: 8-14 (2007)

7. V.R. Tarnawski, T. Momose, W.H. Leong, G. Bovesecchi, P. Coppa, Int. J. Thermo Phys. 30:949 (2009)

8. S.K. Haigh, Géotechnique 62 (7): 617-625 (2012)

9. D. Shrestha, H. Hailemariam, F Wuttke, In Energy Geotechnics-Proceedings of the 1st International Conference on Energy Geotechnics, ICEGT (2016)
10. D. Shrestha, Z.H. Rizvi, F. Wuttke, Heat Mass Transf. 55:1671 (2019)

11. M.A. Shahin, M.B. Jaksa, H.R. Maier, Australian Geomechanics 45 (2002)

12. A.T.C. Goh, J.Geotech. Eng. (1994)

13. Y.M. Najjar \& I.A. Basheer, Geotech Geol. Eng. 14: 193 (1996)

14. M. Grabarczyk, P. Furmanski, J. of Power Tech. 93 (2) 59-66 (2013)

15. Y. Erzin, T. Cetin, Sci Iran 19(2):188-194 (2012)

16. G. Habibagahi, A. Bamdad, Can. Geotech. J. 40 (3): 684-693 (2003)

17. R. Boubou, F. Emeriault, R. Kastner, Can. Geotech. J. 47 (11): 1214-1233 (2010)

18. Z. Hashin, S. Shtrikman, J. Appl. Phys. 33(10):3125-3131 (1962)

19. F. Gori, S. Corasaniti, Planet Space Sci. 52:91-99 (2004)

20. J.C. Maxwell, A treatise on electricity and magnetism, third edn. Dover, New York (1954)

21. V. Balland, P.A. Arp, J. Environ. Eng. Sci. 4(6):549-558 (2005)

22. R.I. Gavriliev, In D.C. Reston (ed.), Thermal analysis, construction, and monitoring methods for frozen ground: 277-294. VA: ASCE (2004) 